\title{
Commissioning tests of the first hydro-generator of the Bajo de Mina HPP (Panama)
}

\author{
Andrey Zuikov ${ }^{1,2, *}$ \\ ${ }^{1}$ Moscow State University of Civil Engineering, Jaroslavskoe Shosse 26, 129337, Moscow, Russia \\ ${ }^{2}$ Moscow Automobile and Road Construction State Technical University, Leningradsky prospect 64, \\ 125319, Moscow, Russia
}

\begin{abstract}
A mathematical model has been developed for calculating the distribution of azimuthal velocities and the shape of the free surface of oil in the compartments of an oil bath of a hydro-generator. The mathematical model of oil flow is verified by comparing the calculated radial-vertical distributions of normalized azimuthal velocities obtained on its basis with the free surface of the oil with the empirical data of third-party authors. The verification showed a good fit between the calculated and experimental distributions, which allows recommending the developed mathematical model for use in engineering practice. Calculations were carried out for the modes of the oil movement in the thrust bearing and the guide bearing of the hydro-generator of the Bajo de Mina HPP. Recommendations are made for the elimination of emergency modes associated with oil overflow through the fencing of the thrust bearing oil bath, which separate oil bath from the shaft of hydro-generator.
\end{abstract}

\section{Introduction}

The first commissioning tests of the Bajo de Mina HPP pioneer hydraulic unit were conducted on July 8, 2011. The unit was abnormally stopped after three minutes of operation at a shaft speed of $200 \mathrm{rpm}$. The reason for the shutdown is the discharge of oil up to 350 liters through the shell (item 2 in Fig. 1,a) of the oil bath of the thrust bearing and the guide bearing to the rotor (item 1 in Fig. 1,a), the poles and the generator winding. The oil level dropped $68 \mathrm{~mm}$ from the normal $915 \mathrm{~mm}$ above the bottom of the oil bath. Actually, this situation led to the need to carry out this work.

Thrust bearings of the vertical-shaft units of hydro power plants are unique mechanical devices that, under tremendous loads, ensure minimal friction between the mirror of the sleeve rotating together with the shaft of the hydraulic unit and the fixed support of the thrust bearing. In essence, the A.E. Aleksandrov's monograph [1] has been remained the only capital work in Russia in this area for many years. The thrust bearing of the Bajo de Mina HPP unit is shown in Fig. 1, where the outlines of the stationary elements are highlighted by a solid black line, and the sleeve rotating together with the shaft at an angular speed $\Omega$ has a gray outline. Because of axial symmetry the drawing is made up to half the section.

The operability of the vertical thrust bearings is ensured by the use of an oil interlayer

* Corresponding author: zuykov54@mail.ru 
("oil wedge") between the rubbing surfaces, which also serves to remove heat $[2,3]$. The dimensions of up-to-date thrust bearings require significant amounts of oil to provide lubrication and cooling of rubbing surfaces $[4,5]$. To place this oil an oil bath is provided in which the thrust bearing is immersed. The oil mass in the bath is in intense circulation [6] excited by the rotation of the sleeve connected to the shaft of the generator. To separate the tank with oil from the hydraulic unit shaft passing through it, a cylindrical shell is arranged (item 2 in Fig. 1,a), which has no hermetic seals with the shaft to eliminate the losses due to friction. The presence of a gap (item 3 in Fig. 1,a) between the shell and the shaft may cause the emergence of oil and its vapors from the oil bath onto the shaft and further onto the parts of the electric generator. This provokes an emergency fire and explosion situation, which is unacceptable and requires immediate remedial measures.

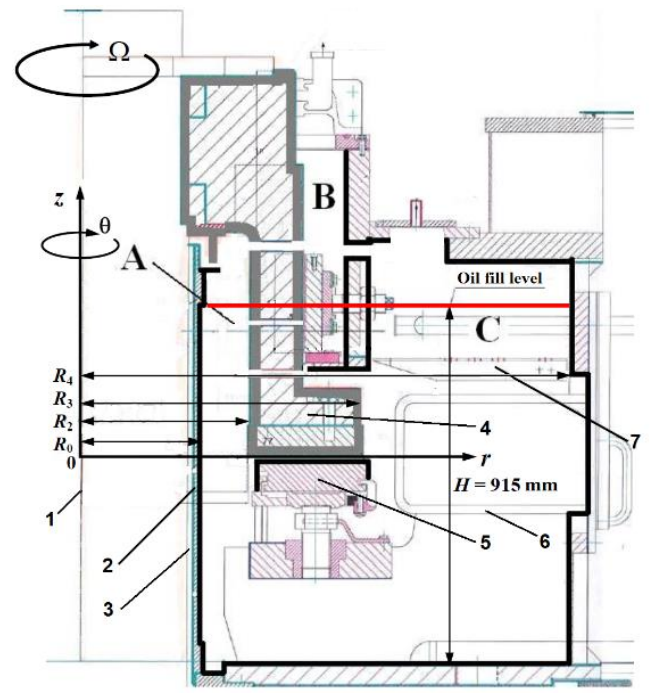

a

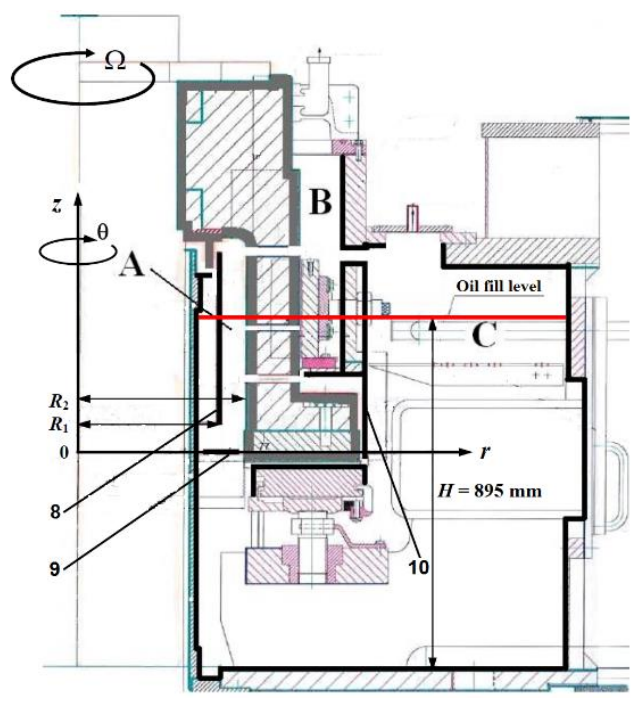

b

Fig. 1. Section through the oil bath of the Bajo de Mina HPP generator thrust bearing: a - oil bath for the moment of supply, $\mathrm{b}$ - modified oil bath; 1 - axis of rotation of the generator shaft, 2 - shell, 3 gap between the shaft and shell, 4 - bush, 5 - thrust bearing support, 6 - cooling system, 7 - sedative shield with grassroots fins, 8 - additional shell, 9 - perforated ring, 10 - annular perforated shield.

Thorough designs of thrust bearings, numerous tests and many years of experience in the design and especially operation of thousands of thrust bearings of vertical-shaft hydraulic units around the world [7 - 16] ensured the creation, effective and reliable operation of these devices, which can be considered one of the most outstanding achievements in the engineering practice.

However, the work of the material at the limit of physical capabilities and sometimes not entirely successful technical solutions lead to emergency situations. Despite the importance of the problem, the technical literature on thrust bearings, their designs, analysis, operation experience, and especially the analysis of cases of failure or emergency situations is quite limited [17 - 22]. Numerous recent developments of the world's leading companies in the field of construction of hydraulic units mainly remain inaccessible for study.

The work was necessitated by the need to eliminate the release of oil through the shell of the thrust bearing oil bath on the shaft of the Bajo de Mina HPP generator, which occurred during the test run of the pioneer hydraulic unit. The cause of the ejection seems to be the result of a significant distortion of the free surface of the oil from horizontal due to its intensive circulation movement in compartment A (cf. Fig. 1) between the stationary shell and the rotating thrust bearing bush. In this regard, the task was set to carry out analytical 
studies of distribution of azimuthal oil velocities in the thrust bearing bath, focusing on compartment A. Based on the found azimuthal velocity distributions, calculate the shape of the oil free surface and to give recommendations on changes in the design of the oil bath in order to correct the fire and explosion emergency situation.

\section{Method}

Research method is analytical. For analytical calculation of the oil circulation movement in the compartments of the oil bath of the hydro-generator thrust bearing and guide bearing the Navier - Stokes equations in a cylindrical coordinate system are taken as the initial ones [6]

$$
\left.\begin{array}{c}
\frac{\partial u_{r}}{\partial t}+u_{r} \frac{\partial u_{r}}{\partial r}+u_{\theta} \frac{\partial u_{r}}{r \partial \theta}+u_{z} \frac{\partial u_{r}}{\partial z}-\frac{u_{\theta}^{2}}{r}= \\
=-\frac{\partial}{\partial r}\left(\frac{P}{\rho}-\Pi\right)+v\left(\nabla^{2} u_{r}-\frac{u_{r}}{r^{2}}-2 \frac{\partial u_{\theta}}{r^{2} \partial \theta}\right), \\
\frac{\partial u_{\theta}}{\partial t}+u_{r} \frac{\partial u_{\theta}}{\partial r}+u_{\theta} \frac{\partial u_{\theta}}{r \partial \theta}+u_{z} \frac{\partial u_{\theta}}{\partial z}+u_{r} \frac{u_{\theta}}{r}= \\
=-\frac{\partial}{r \partial \theta}\left(\frac{P}{\rho}-\Pi\right)+v\left(\nabla^{2} u_{\theta}-\frac{u_{\theta}}{r^{2}}+2 \frac{\partial u_{r}}{r^{2} \partial \theta}\right), \\
\frac{\partial u_{z}}{\partial t}+u_{r} \frac{\partial u_{z}}{\partial r}+u_{\theta} \frac{\partial u_{z}}{r \partial \theta}+u_{z} \frac{\partial u_{z}}{\partial z}=-\frac{\partial}{\partial z}\left(\frac{P}{\rho}-\Pi\right)+v \nabla^{2} u_{z},
\end{array}\right\}
$$

where $r, \theta, z$ are radial, azimuthal and vertical coordinates; $t$ is the current time; $u_{r}, u_{\theta}, u_{z}$ are radial, azimuthal and vertical components of the oil velocity; $\rho, v$ are oil density and viscosity; $P, \Pi$ are pressure and potential of external mass forces; $\nabla^{2} u_{i}$ is Laplacian.

\section{Results and Discussion}

\subsection{Analysis of emergency situation}

In the mode of operation of a hydraulic unit, the oil movement in compartments A, B, C of the oil bath can be assumed as steady-state and having symmetry with respect to vertical axis z. This allows one to exclude partial derivatives with respect to time and azimuthal coordinate. Further, assuming radial and vertical velocities are negligible in comparison with azimuthal $\left(u_{r} \ll<u_{\theta}, u_{z} \ll<u_{\theta}\right)$, system (1) is reduced to.

$$
\left.\begin{array}{l}
\frac{\partial}{\partial r}\left(\frac{P}{\rho}-\Pi\right)=\frac{u_{\theta}^{2}}{r} \\
\frac{\partial^{2} u_{\theta}}{\partial r^{2}}+\frac{\partial u_{\theta}}{r \partial r}-\frac{u_{\theta}}{r^{2}}+\frac{\partial^{2} u_{\theta}}{\partial z^{2}}=0 \\
\frac{\partial}{\partial z}\left(\frac{P}{\rho}-\Pi\right)=0
\end{array}\right\}
$$

The analysis of the equations of system (2) shows that for suppressing the significant distortion of the shape of the oil free surface in compartment $\mathrm{A}$ it is necessary to reduce the 
thickness of the rotating layer between the shell and the bush and suppress the rotational movement of the oil in compartment $\mathrm{C}$. Based on these considerations, a modification of the oil bath is proposed, shown in Fig. 1,b. Unlike the factory-supplied oil bath (cf. Fig. 1,a) in a modified bath:

- an additional shell is made in compartment A (item 8 in Fig. 1,b), as a result of which the thickness of the rotating oil layer between the shell and the bush is halved leading to suppression of a significant increment in the level of the free surface to the inner wall of the rotating bush;

- a perforated ring is installed in compartment A (item 9 in Fig. 1,b), which inhibits the nearest oil layers, which also serves to reduce the difference in the oil free surface level between the wall of the additional shell on radius $R_{1}$ and the inner wall of the bush on radius $R_{2}$;

- compartment $\mathrm{C}$ is equipped with an annular perforated shield (item 10 in Fig. 1,b) closing the compartment from the rotating bush;

- the oil filling level is reduced from 915 to $895 \mathrm{~mm}$.

It can be seen that in the modified oil bath, the oil circulating movement will be only in compartment A. Complicated movement will be observed in compartment B, because stable rotation of the oil cannot be formed here, which is prevented by the guide bearing segments. Such flows cannot be correctly analytically described. However, compartment B is a closed cavity, in which the oil movement is unable to influence the oil movement in other compartments. In compartment $\mathrm{C}$ there will be a slight movement of oil suppressed by annular and ribbed sedative shields, as well as by the pipes of the cooling system. In general, there is hydrostatics here.

\subsection{Model of oil movement in compartment $\mathrm{A}$ in thrust bearing oil bath}

Normalizing the second equation of system (2) according to the inner radius of bush $R_{2}$ in compartment A (cf. Fig. 1) and the angular velocity of rotation of the hydraulic unit $\Omega$, putting in it

$$
\dot{r}=\frac{r}{R_{2}}, \quad \dot{z}=\frac{z}{R_{2}}, \quad \dot{u}_{\theta}=\frac{u_{\theta}}{\Omega R_{2}},
$$

we obtain a dimensionless equation of the form

$$
\frac{\partial^{2} \dot{u}_{\theta}}{\partial \dot{r}^{2}}+\frac{\partial \dot{u}_{\theta}}{\dot{r} \partial \dot{r}}-\frac{\dot{u}_{\theta}}{\dot{r}^{2}}+\frac{\partial^{2} \dot{u}_{\theta}}{\partial \dot{z}^{2}}=0
$$

The boundary conditions of the problem are as follows:

- the azimuthal oil velocities equal to zero $\left(\dot{u}_{\theta}=0\right)$ on the fixed walls of the primary (item 2 in Fig. 1,a) and additional (item 8 in Fig. 1,b) shells at normalized radii, respectively $\dot{r}_{0}=R_{0} / R_{2}$ and $\dot{r}_{1}=R_{1} / R_{2}$

- equality of azimuthal oil velocities to peripheral velocities of the inner surface of the rotating bush $\left(\dot{u}_{\theta}=1\right)$ at a normalized radius $\dot{r}_{2}=R_{2} / R_{2}=1$ (item 4 in Fig. 1,a);

- the azimuthal oil velocities equal to zero $\left(\dot{u}_{\theta}=0\right)$ on the surface of the perforated ring in compartment A at the level $\dot{z}=0$ (item 9 in Fig. 1,b).

So, function $\dot{u}_{\theta}(\dot{r}, \dot{z})$ has the following boundary conditions: 


$$
\left.\begin{array}{l}
\dot{u}_{\theta}\left(\dot{r}_{0}, \dot{z}\right)=\dot{u}_{\theta}\left(\dot{r}_{1}, \dot{z}\right)=0, \\
\dot{u}_{\theta}(1, \dot{z})=1 \\
\dot{u}_{\theta}(\dot{r}, 0)=0 .
\end{array}\right\}
$$

In equation (4), we expand function $\dot{u}_{\theta}(\dot{r}, \dot{z})$ by Fourier, i.e. put

$$
\dot{u}_{\theta}(\dot{r}, \dot{z})=\phi(\dot{r}) \cdot \varphi(\dot{z})
$$

where one of the factors $\phi=\phi(\dot{r})$ depends only on the current radius, and the second one $\varphi=\varphi(\dot{z})-$ only on the vertical coordinate.

Then, substituting (6) in (4), we find

$$
\varphi\left(\frac{\partial^{2} \phi}{\partial \dot{r}^{2}}+\frac{\partial \phi}{\dot{r} \partial \dot{r}}-\frac{\phi}{\dot{r}^{2}}\right)+\phi \frac{\partial^{2} \varphi}{\partial \dot{z}^{2}}=0,
$$

dividing this expression further by $\phi \varphi$ and separating the variables, we obtain

$$
\frac{1}{\varphi} \cdot \frac{\partial^{2} \varphi}{\partial \dot{z}^{2}}=-\frac{1}{\phi}\left(\frac{\partial^{2} \phi}{\partial \dot{r}^{2}}+\frac{\partial \phi}{\dot{r} \partial \dot{r}}-\frac{\phi}{\dot{r}^{2}}\right) .
$$

If the left side of the resulting equality is a product of functions that depend only on variable $\dot{z}$, and the right one depends only on $\dot{r}$, then it can be satisfied in the only case - if neither its left nor right sides depend neither on $\dot{z}$, nor on $\dot{r}$, i.e. both sides are equal to the same separation constant. Let us denote it by $\eta$. Then,

$$
\frac{1}{\varphi} \cdot \frac{\partial^{2} \varphi}{\partial \dot{z}^{2}}=-\frac{1}{\phi}\left(\frac{\partial^{2} \phi}{\partial \dot{r}^{2}}+\frac{\partial \phi}{\dot{r} \partial \dot{r}}-\frac{\phi}{\dot{r}^{2}}\right)=\eta .
$$

This allows us to decompose (7) into a system of two linear differential equations

$$
\left.\begin{array}{l}
\frac{\partial^{2} \varphi}{\partial \dot{z}^{2}}-\eta \varphi=0, \\
\frac{\partial^{2} \phi}{\partial \dot{r}^{2}}+\frac{\partial \phi}{\dot{r} \partial \dot{r}}+\left(\eta-\frac{1}{\dot{r}^{2}}\right) \phi=0
\end{array}\right\}
$$

and reduce the desired solution to the Sturm-Liouville eigenvalue problem: to find such values of parameter $\eta$ with which there exist identically non-zero solutions of equations (8) satisfying the boundary conditions (5).

The equations (8) has three solutions: the first for $\eta>0$, the second for $\eta=0$ and the third one for $\eta<0$.

In the first case, let $\eta=\lambda_{n}^{2}>0$, then, 


$$
\left.\begin{array}{l}
\frac{\partial^{2} \varphi}{\partial \dot{z}^{2}}-\lambda_{n}^{2} \varphi=0 \\
\frac{\partial^{2} \phi}{\partial \dot{r}^{2}}+\frac{\partial \phi}{\dot{r} \partial \dot{r}}+\left(\lambda_{n}^{2}-\frac{1}{\dot{r}^{2}}\right) \phi=0
\end{array}\right\}
$$

The first equation of system (9) has the following solution

$$
\varphi_{n}(\dot{z})=C_{1} \exp \left(\lambda_{n} \dot{z}\right)+C_{2} \exp \left(-\lambda_{n} \dot{z}\right)
$$

while the second one relates to Bessel equations [23]

$$
\frac{d^{2} \phi_{n}}{d \dot{r}^{2}}+(1-2 a) \frac{d \phi_{n}}{\dot{r} d \dot{r}}+\left[\left(\lambda_{n} c \dot{r}^{c-1}\right)^{2}+\frac{a^{2}-m^{2} c^{2}}{\dot{r}^{2}}\right] \phi_{n}=0
$$

whose particular solution has the form

$$
\phi_{n}(\dot{r})=A_{n} \dot{r}^{a} J_{m}\left(\lambda_{n} \dot{r}^{c}\right),
$$

where $A_{n}$ is the coefficient (constant) of the $n$-th particular solution, $J_{m}\left(\lambda_{n} \dot{r}^{c}\right)$ is the cylindrical Bessel function of the first kind of the $m$-th order.

Hence, according to (9) - (11)

$$
\phi_{n}(\dot{r})=A_{n} J_{1}\left(\lambda_{n} \dot{r}\right)
$$

where $J_{1}\left(\lambda_{n} \dot{r}\right)$ is the Bessel function of the first kind of the first order.

Using the complete system of particular solutions, according to (6) we obtain the FourierBessel series

$$
\dot{u}_{\theta}(\dot{r}, \dot{z})=\phi(\dot{r}) \cdot \varphi(\dot{z})=\sum_{n=1}^{\infty} A_{n} J_{1}\left(\lambda_{n} \dot{r}\right)\left[C_{1} \exp \left(\lambda_{n} \dot{z}\right)+C_{2} \exp \left(-\lambda_{n} \dot{z}\right)\right]
$$

In the solution obtained, constant $C_{1}$ should be set equal to zero, because in the opposite case, the azimuthal velocities would have to rise to $\dot{u}_{\theta} \rightarrow \pm \infty$ at $\dot{z} \rightarrow \infty$ and let constant $C_{2}$ be included in $A_{n}$. Hence, for the case $\eta>0$, we have

$$
\dot{u}_{\theta}(\dot{r}, \dot{z})=\phi(\dot{r}) \cdot \varphi(\dot{z})=\sum_{n=1}^{\infty} A_{n} J_{1}\left(\lambda_{n} \dot{r}\right) \exp \left(-\lambda_{n} \dot{z}\right) .
$$

In the second case with $\eta=0$ we find

$$
\left.\begin{array}{l}
\frac{\partial^{2} \varphi}{\partial \dot{z}^{2}}=0, \\
\frac{\partial^{2} \phi}{\partial \dot{r}^{2}}+\frac{\partial \phi}{\dot{r} \partial \dot{r}}-\frac{\phi}{\dot{r}^{2}}=0
\end{array}\right\}
$$

from where 


$$
\left.\begin{array}{l}
\varphi(\dot{z})=C_{3} \dot{z}+C_{4}, \\
\phi(\dot{r})=C_{5} \dot{r}+\frac{C_{6}}{\dot{r}}
\end{array}\right\}
$$

and

$$
\dot{u}_{\theta}(\dot{r}, \dot{z})=\phi(\dot{r}) \cdot \varphi(\dot{z})=\left(C_{5} \dot{r}+\frac{C_{6}}{\dot{r}}\right)\left(C_{3} \dot{z}+C_{4}\right) .
$$

Distribution of azimuthal velocities according to (13) is known as the Couette flow. Such a distribution takes place during static rotation of a fluid or in the gap between rotating coaxial cylinders [24], including, as in the case under study, when one of them is stationary. In (13), for the reasons stated above, we take $C_{3}=0$ and $C_{4}=1$. As a result, with $\eta=0$, we find

$$
\dot{u}_{\theta}(\dot{r})=C_{5} \dot{r}+\frac{C_{6}}{\dot{r}} .
$$

In the third case with $\eta=-\lambda_{n}^{2}<0$ system (8) is reduced to the form

$$
\left.\begin{array}{l}
\frac{\partial^{2} \varphi}{\partial \dot{z}^{2}}+\lambda_{n}^{2} \varphi=0 \\
\frac{\partial^{2} \phi}{\partial \dot{r}^{2}}+\frac{\partial \phi}{\dot{r} \partial \dot{r}}-\left(\lambda_{n}^{2}+\frac{1}{\dot{r}^{2}}\right) \phi=0 .
\end{array}\right\}
$$

It can be seen that the second equation (15) is a modified Bessel equation [23] that does not have real roots. Therefore, case $\eta<0$ is excluded from further consideration.

Thus, the general solution to the problem is the sum of (12) and (14), since the sum of the solutions of a system of linear differential equations is also its solution

$$
\dot{u}_{\theta}(\dot{r}, \dot{z})=\phi(\dot{r}) \cdot \varphi(\dot{z})=C_{5} \dot{r}+\frac{C_{6}}{\dot{r}}+\sum_{n=1}^{\infty} A_{n} J_{1}\left(\lambda_{n} \dot{r}\right) \exp \left(-\lambda_{n} \dot{z}\right) .
$$

The component of the general distribution (16), given by the Couette radial profile according to (14), is the final solution to the problem with $\dot{z} \rightarrow \infty$ because in this case the third term containing $\exp \left(-\lambda_{n} \dot{z}\right)$ vanishes. This solution satisfies the first two boundary conditions (5), determining by which the integration constants $C_{5}$ and $C_{6}$, we obtain

$$
\dot{u}_{\theta}(\dot{r})=\frac{\dot{r}}{1-\dot{r}_{0}^{2}}\left(1-\frac{\dot{r}_{0}^{2}}{\dot{r}^{2}}\right) \quad \text { or } \quad \dot{u}_{\theta}(\dot{r})=\frac{\dot{r}}{1-\dot{r}_{1}^{2}}\left(1-\frac{\dot{r}_{1}^{2}}{\dot{r}^{2}}\right) \text {. }
$$

The normalized distributions (17) give $\dot{u}_{\theta}(\dot{r})=0$ with $\dot{r}=\dot{r}_{0}$ or $\dot{r}=\dot{r}_{1}$, i.e. on the stationary walls of the main (item 2 in Fig. 1,a) and additional (item 8 in Fig. 1,b) shells, and $\dot{u}_{\theta}(\dot{r})=1$ on the surface of the rotating generator thrust bearing bush with $\dot{r}=\dot{r}_{2}=1$.

Substituting (17) in (16) we find

$$
\dot{u}_{\theta}(\dot{r}, \dot{z})=\phi(\dot{r}) \cdot \varphi(\dot{z})=\frac{\dot{r}}{1-\dot{r}_{0}^{2}}\left(1-\frac{\dot{r}_{0}^{2}}{\dot{r}^{2}}\right)+\sum_{n=1}^{\infty} A_{n} J_{1}\left(\lambda_{n} \dot{r}\right) \exp \left(-\lambda_{n} \dot{z}\right),
$$




$$
\dot{u}_{\theta}(\dot{r}, \dot{z})=\phi(\dot{r}) \cdot \varphi(\dot{z})=\frac{\dot{r}}{1-\dot{r}_{1}^{2}}\left(1-\frac{\dot{r}_{1}^{2}}{\dot{r}^{2}}\right)+\sum_{n=1}^{\infty} A_{n} J_{1}\left(\lambda_{n} \dot{r}\right) \exp \left(-\lambda_{n} \dot{z}\right)
$$

Solutions (18) satisfy all boundary conditions (5) if $\lambda_{n}$ is one of the real zeros of the firstorder Bessel function of the first kind $\left(J_{1}\left(\lambda_{n}\right)=0\right)$, which includes constants $\dot{r}_{0}$ or $\dot{r}_{1}$ as the factors, because with $\dot{u}_{\theta}\left(\dot{r}_{0}, \dot{z}\right)=\dot{u}_{\theta}\left(\dot{r}_{1}, \dot{z}\right)=0$, simultaneously $\dot{u}_{\theta}(\dot{r}, \dot{z})=1$ with $\dot{r}=\dot{r}_{2}=1$ and also $\dot{u}_{\theta}(\dot{r}, \dot{z})=0$ with $\dot{z}=0$ that according to (18), it is ensured by equalities

$$
\begin{aligned}
& \frac{\dot{r}}{1-\dot{r}_{0}^{2}}\left(1-\frac{\dot{r}_{0}^{2}}{\dot{r}^{2}}\right)=-\sum_{n=1}^{\infty} A_{n} J_{1}\left(\lambda_{n} \dot{r}\right) \text { or } \\
& \frac{\dot{r}}{1-\dot{r}_{1}^{2}}\left(1-\frac{\dot{r}_{1}^{2}}{\dot{r}^{2}}\right)=-\sum_{n=1}^{\infty} A_{n} J_{1}\left(\lambda_{n} \dot{r}\right) .
\end{aligned}
$$

The solution of equations (19) is reduced to finding the values of constants $A_{n}$ satisfying these equalities. For this, we use the formal conditions of orthogonally of Bessel functions [23], according to which: if $\lambda_{i}$ and $\lambda_{j}$ are two zeros of function $J_{m}\left(\lambda_{n}\right)=0$ are real, then,

$$
\left.\begin{array}{ll}
\int_{0}^{1} J_{m}\left(\lambda_{i} \dot{r}\right) J_{m}\left(\lambda_{j} \dot{r}\right) \dot{r} d \dot{r}=0, & \text { if } \quad i \neq j, \\
\int_{0}^{1} J_{m}\left(\lambda_{i} \dot{r}\right) J_{m}\left(\lambda_{j} \dot{r}\right) \dot{r} d \dot{r}=\frac{1}{2}\left[J_{m}^{\prime}\left(\lambda_{i}\right)\right]^{2}, & \text { if } \quad i=j .
\end{array}\right\}
$$

Then, multiplying the right and left sides of the first equation (19) sequentially by $\dot{r} J_{1}\left(\lambda_{1} \dot{r}\right) d \dot{r}, \dot{r} J_{1}\left(\lambda_{n} \dot{r}\right) d \dot{r}$ and further to $\dot{r} J_{1}\left(\lambda_{\infty} \dot{r}\right) d \dot{r}$ when integrating over the interval $\dot{r}$ from 0 to 1 , we obtain the system of equalities

$$
\begin{gathered}
\frac{1}{1-\dot{r}_{0}^{2}} \int_{0}^{1} \dot{r}^{2} J_{1}\left(\lambda_{1} \dot{r}\right) d \dot{r}-\frac{\dot{r}_{0}^{2}}{1-\dot{r}_{0}^{2}} \int_{0}^{1} J_{1}\left(\lambda_{1} \dot{r}\right) d \dot{r}=-A_{1} \int_{0}^{1} J_{1}\left(\lambda_{1} \dot{r}\right) J_{1}\left(\lambda_{1} \dot{r}\right) \dot{r} d \dot{r}, \\
\frac{1}{1-\dot{r}_{0}^{2}} \int_{0}^{1} \dot{r}^{2} J_{1}\left(\lambda_{2} \dot{r}\right) d \dot{r}-\frac{\dot{r}_{0}^{2}}{1-\dot{r}_{0}^{2}} \int_{0}^{1} J_{1}\left(\lambda_{2} \dot{r}\right) d \dot{r}=-A_{2} \int_{0}^{1} J_{1}\left(\lambda_{2} \dot{r}\right) J_{1}\left(\lambda_{2} \dot{r}\right) \dot{r} d \dot{r}, \\
\frac{1}{1-\dot{r}_{0}^{2}} \int_{0}^{1} \dot{r}^{2} J_{1}\left(\lambda_{n} \dot{r}\right) d \dot{r}-\frac{\dot{r}_{0}^{2}}{1-\dot{r}_{0}^{2}} \int_{0}^{1} J_{1}\left(\lambda_{n} \dot{r}\right) d \dot{r}=-A_{n} \int_{0}^{1} J_{1}\left(\lambda_{n} \dot{r}\right) J_{1}\left(\lambda_{n} \dot{r}\right) \dot{r} d \dot{r}, \\
\frac{1}{1-\dot{r}_{0}^{2}} \int_{0}^{1} \dot{r}^{2} J_{1}\left(\lambda_{\infty} \dot{r}\right) d \dot{r}-\frac{\dot{r}_{0}^{2}}{1-\dot{r}_{0}^{2}} \int_{0}^{1} J_{1}\left(\lambda_{\infty} \dot{r}\right) d \dot{r}=-A_{\infty} \int_{0}^{1} J_{1}\left(\lambda_{\infty} \dot{r}\right) J_{1}\left(\lambda_{\infty} \dot{r}\right) \dot{r} d \dot{r} .
\end{gathered}
$$

Whence for an arbitrary $n$-th particular solution as a result of integration we find 


$$
\frac{1}{1-\dot{r}_{0}^{2}} \cdot \frac{J_{2}\left(\lambda_{n}\right)}{\lambda_{n}}-\frac{\dot{r}_{0}^{2}}{1-\dot{r}_{0}^{2}} \cdot \frac{1-J_{0}\left(\lambda_{n}\right)}{\lambda_{n}}=-\frac{A_{n}}{2}\left[J_{1}^{\prime}\left(\lambda_{n}\right)\right]^{2},
$$

where $J_{0}\left(\lambda_{n}\right)$ and $J_{2}\left(\lambda_{n}\right)$ are the Bessel functions of the first kind of zero and second orders.

We now use the recurrence relations [23] connecting the cylindrical functions of various orders to each other

$$
J_{m+1}(x)=\frac{2 m}{x} J_{m}(x)-J_{m-1}(x)=\frac{m}{x} J_{m}(x)-\frac{d}{d x} J_{m}(x)=-x^{m} \frac{d}{d x}\left[x^{-m} J_{m}(x)\right],
$$

here $x$ is the argument of cylindrical function.

According to the recurrence relations, we can see that with $J_{1}\left(\lambda_{n}\right)=0$ the equalities

$$
J_{1}^{\prime}\left(\lambda_{n}\right)=J_{0}\left(\lambda_{n}\right), \quad J_{2}\left(\lambda_{n}\right)=-J_{0}\left(\lambda_{n}\right),
$$

substituting thereof in relation (20), we obtain

$$
A_{n}=\frac{2}{\left(1-\dot{r}_{0}^{2}\right) \lambda_{n} J_{0}\left(\lambda_{n}\right)}\left(1-\dot{r}_{0}^{2}\left[1-\frac{1}{J_{0}\left(\lambda_{n}\right)}\right]\right) .
$$

For the second equation (19) we obtain similar relationship

$$
A_{n}=\frac{2}{\left(1-\dot{r}_{1}^{2}\right) \lambda_{n} J_{0}\left(\lambda_{n}\right)}\left(1-\dot{r}_{1}^{2}\left[1-\frac{1}{J_{0}\left(\lambda_{n}\right)}\right]\right) .
$$

Introducing constants $A_{n}$ into Eqs. (18), we finally write down the radial-vertical distribution of normalized azimuthal velocities in compartment A of the oil bath of the hydrogenerator thrust bearing.

$$
\begin{aligned}
& \dot{u}_{\theta}(\dot{r}, \dot{z})=\frac{1}{1-\dot{r}_{0}^{2}}\left\{\dot{r}-\frac{\dot{r}_{0}^{2}}{\dot{r}}+2 \sum_{n=1}^{\infty} \frac{J_{1}\left(\lambda_{n} \dot{r}\right)}{\lambda_{n} J_{0}\left(\lambda_{n}\right)}\left(1-\dot{r}_{0}^{2}\left[1-\frac{1}{J_{0}\left(\lambda_{n}\right)}\right]\right) \exp \left(-\lambda_{n} \dot{z}\right)\right\}, \\
& \dot{u}_{\theta}(\dot{r}, \dot{z})=\frac{1}{1-\dot{r}_{1}^{2}}\left\{\dot{r}-\frac{\dot{r}_{1}^{2}}{\dot{r}}+2 \sum_{n=1}^{\infty} \frac{J_{1}\left(\lambda_{n} \dot{r}\right)}{\lambda_{n} J_{0}\left(\lambda_{n}\right)}\left(1-\dot{r}_{1}^{2}\left[1-\frac{1}{J_{0}\left(\lambda_{n}\right)}\right]\right) \exp \left(-\lambda_{n} \dot{z}\right)\right\} .
\end{aligned}
$$

To convert the normalized values of the azimuthal oil velocities according to (21) to absolute ones, it is necessary to use the normalizing relations (3).

Note that according to (21), distribution of azimuthal velocities does not depend on the physical properties of the fluid. Therefore, at any density and viscosity of oil or modeling fluid (for example, water), the distribution of azimuthal velocities will remain unchanged. Physical modeling of such flows should be performed according to the Froude criterion.

Verification of the azimuthal oil velocities distribution model in compartment A was carried out according to the experimental data of the research work [25]. This work presents physical studies of circulating movement of oil in compartment A on a laboratory model of the thrust bearing oil bath of the Jinvali hydropower plant (Georgia) where same generators with the same oil bath are installed and same problems as on the Bajo de Mina HPP. The purpose of the work is to identify on the physical model the causes of oil overflow through the shell of the thrust bearing oil bath on the hydro-generator shaft. Thus, for the Jinvali 
hydropower plant, the same problem was solved in the research process as in the present study. The scale of the model was $\mathrm{M}=1: 2.9$; the modeling fluid was water; the rotating cylinder simulating the bush had an internal radius of $R_{2}=147.5 \mathrm{~mm}$; a stationary cylinder simulating the shell had a radius of $R_{0}=126 \mathrm{~mm}$ and could be replaced by cylinders of radii $R_{l}=133.5 \mathrm{~mm}$ and $R_{1}=141.5 \mathrm{~mm}$; the bush rotation speed ranged from 163 to $750 \mathrm{rpm}$. In the research process the precision measuring equipment was presented by laser Doppler liquid velocity meter.

As the result of the studies the curve obtained describes the distribution of azimuthal oil velocities in the bottom layers in compartment $A$ of the thrust bearing oil bath of the generator. This curve with experimental points is shown in Fig. 2,a and is designated with index " $E$ " (experiment). Simultaneously Fig. 2,a, shows the results of calculations of distribution of normalized azimuthal oil velocities over the height and radius of compartment A. performed in accordance with dependence (21). In the calculations in accordance with the model parameters accepted was $\dot{r}_{0}=R_{0} / R_{2}=126 / 147.5=0.854$.

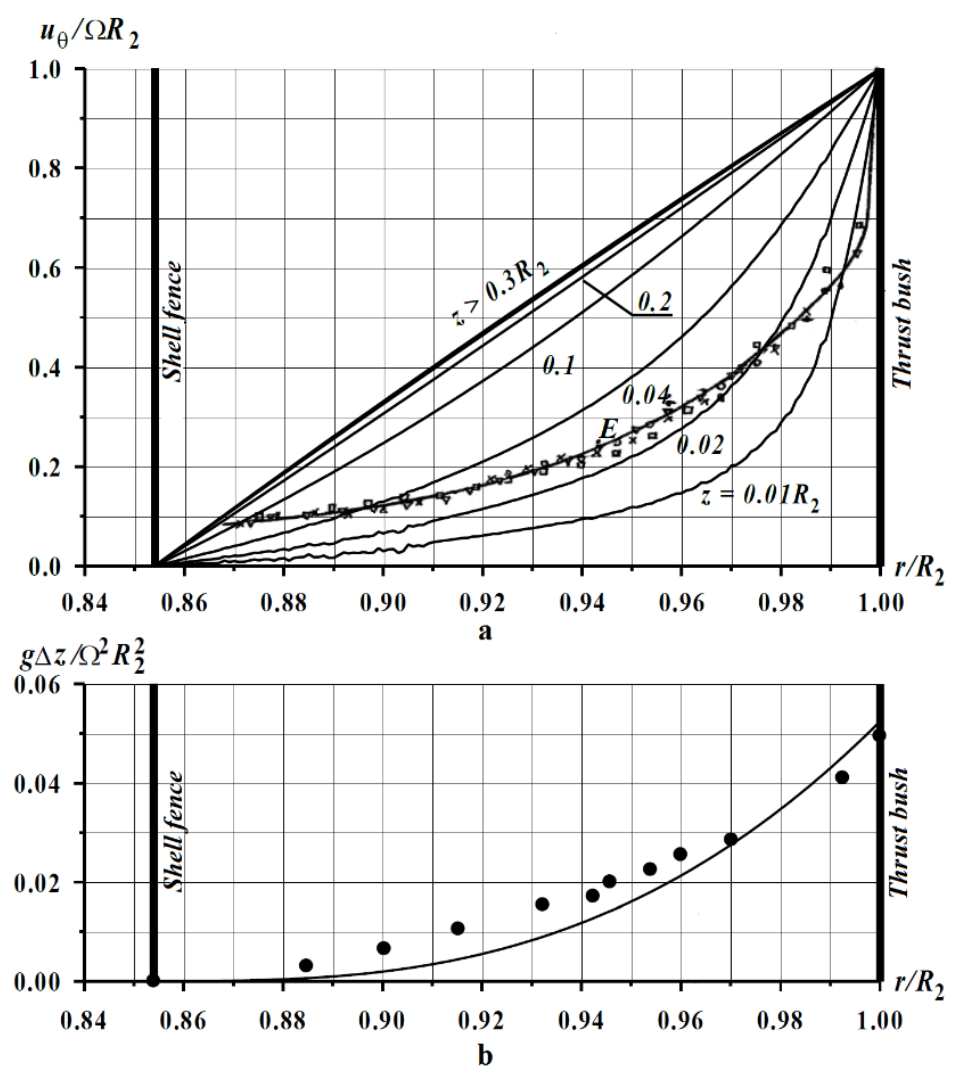

Fig. 2. Design and experimental characteristics of oil movement in compartment A of the generator thrust bearing oil bath: a - distribution of azimuthal velocities by the height and radius of the compartment, $b$ - curve of the free surface.

Based on the results of verification calculations, the following conclusions can be drawn: - the calculated distribution curves of normalized azimuthal oil velocities by the radius in the near-bottom layers of compartment $\mathrm{A}$ of the oil bath satisfactorily correspond to the experimental data;

- at a height exceeding $z=0.3 R_{2}$, the braking effect of the bottom ceases to affect the azimuthal velocities diagram and their distribution is described by formulas (17). 
With the known distribution of azimuthal velocities, the free surface of the oil can be determined from the first and third equations of system (2), in which the potential of external mass forces under gravity with acceleration $g=9.81 \mathrm{~m} / \mathrm{sec}^{2}$ is equal to

$$
\Pi=-g z \text {. }
$$

Multiplying the first equation (2) by $d r$, and the third one by $d z$, respectively, and adding them, we find

$$
\frac{\partial}{\partial r}\left(\frac{P}{\rho}+g z\right) d r+\frac{\partial}{\partial z}\left(\frac{P}{\rho}+g z\right) d z=\frac{u_{\theta}^{2}}{r} d r
$$

The expression of the first side of the equation is the total differential equation wherefrom

$$
d\left(\frac{P}{\rho}+g z\right)=\frac{u_{\theta}^{2}}{r} d r .
$$

One of the surfaces of equal pressure that meets condition $d P=0$ is the free surface of the oil, the radial change of which is determined by differential equation

$$
d z=\frac{1}{g} \cdot \frac{u_{\theta}^{2}}{r} d r
$$

To solve equation (22), we pay attention to the following points. The recommended level of oil filling into the modernized bath of the thrust bearing of the Bajo de Mina HPP hydro generator is $H=895 \mathrm{~mm}$ above its bottom. This corresponds to the level of the free surface of oil at rest (at $\Omega=0$ ) above the perforated ring (item 9 in Fig. 1,b) equal to $z_{0}=270 \mathrm{~mm}$. If the radius of the inner surface of the thrust bearing bush is equal to $R_{2}=430 \mathrm{~mm}$, then the ratio $z_{0} / R_{2}$ will be 0.628 . This exceeds the value $z / R_{2}=0.3$, above which the distribution of azimuthal velocities depends only on the current radius $r$. The ratio $z_{0} / R_{2}>0.3$ is characteristic of all thrust bearings of vertical-shaft hydraulic units. Therefore, the calculation of the radial change in the level of the free surface of the oil in compartment A can be performed using formula (17), which in dimensional form takes the form

$$
\begin{gathered}
u_{\theta}(r)=\frac{\Omega R_{2}^{2}}{R_{2}^{2}-R_{0}^{2}}\left(r-\frac{R_{0}^{2}}{r}\right) \text { or } \\
u_{\theta}(r)=\frac{\Omega R_{2}^{2}}{R_{2}^{2}-R_{1}^{2}}\left(r-\frac{R_{1}^{2}}{r}\right) .
\end{gathered}
$$

To determine the height position of the oil free surface curve above the perforated ring it is necessary to know the value of its ordinate $z$ at the shell (item 2 in Fig. 1,a). Considering that in compartment $\mathrm{C}$ the oil free surface area is much larger than the oil free surface area in compartment $\mathrm{A}$, we assume that the value of this ordinate negligibly differs from the level of the oil free surface in the oil bath at rest $z_{0}$. Then, integrating (22) on the left side from $z_{0}$ to $z$, and on the right side from $R_{0}$ or $R_{1}$ to $R_{2}$, taking into account the above, we find

$$
\Delta z=z-z_{0}=\frac{1}{g} \cdot\left(\frac{\Omega R_{2}^{2}}{R_{2}^{2}-R_{0}^{2}}\right)^{2}\left[\frac{r^{2}}{2}\left(1-\frac{R_{0}^{4}}{r^{4}}\right)-2 R_{0}^{2} \ln \left(\frac{r}{R_{0}}\right)\right],
$$




$$
\Delta z=z-z_{0}=\frac{1}{g} \cdot\left(\frac{\Omega R_{2}^{2}}{R_{2}^{2}-R_{1}^{2}}\right)^{2}\left[\frac{r^{2}}{2}\left(1-\frac{R_{1}^{4}}{r^{4}}\right)-2 R_{1}^{2} \ln \left(\frac{r}{R_{1}}\right)\right]
$$

where $z$ is the level of the oil free surface at the current radius $r ; \Delta z$ is the excess of level $z$ over $z_{0}$.

Verification of the calculation of the shape of the oil free surface in compartment A was made based on experimental data of the above research work [25]. In Fig. 2,b, the experimental data are shown by dots; the calculation in accordance with (24) is shown by a solid line.

Based on the results of the verification calculation the following conclusions can be drawn:

- the calculated curve of the oil free surface along the radius of compartment A satisfactorily agrees with the experimental data;

- the shape of the oil free surface in compartment A is close to the paraboloid of revolution.

The verification of the method for calculating the azimuthal velocities and oil free surface in compartment $\mathrm{A}$ of the thrust bearing oil bath substantiates the application of the method in the engineering practice.

\subsection{Analysis of oil movement parameters in thrust bearing oil bath}

At the time of delivery, the oil bath of the thrust bearing and the upper guide bearing of the Bajo de Mina HPP generator corresponded to Fig. 1,a; its technical characteristics are given in Table 1.

Table 1. Characteristics of oil bath of thrust bearing of Bajo de Mina HPP generator.

\begin{tabular}{|l|c|l|c|}
\hline Rated speed of shaft rotation & $450 \mathrm{rpm}$ & Outer bush face radius $R_{3}$ & $710 \mathrm{~mm}$ \\
\hline Shell radius $R_{0}$ & $300 \mathrm{~mm}$ & Oil bath radius $R_{4}$ & $1020 \mathrm{~mm}$ \\
\hline Inner bush radius $R_{2}$ & $430 \mathrm{~mm}$ & Oil filling level & $915 \mathrm{~mm}$ \\
\hline
\end{tabular}

As noted at the beginning of the article, during a test run and setting the shaft speed by the hydraulic unit of $200 \mathrm{rpm}$, which corresponds to the angular shaft rotation speed of $\Omega=$ $20.94 \mathrm{rad} / \mathrm{sec}$, oil was ejected from the oil bath of the thrust bearing and upper guide bearing through the shell to the hydro-generator shaft. The unit was abnormally shut-down after three minutes of operation. The volume of oil overflown trapped on the rotor, the poles and the winding of the generator, amounted to 300 - 350 liters. The oil level decreased by $68 \mathrm{~mm}$ from the nominal $915 \mathrm{~mm}$ above the bottom of the oil bath.

Fig. 3 shows the design charts of distribution of azimuthal velocities $\left(u_{\theta}\right)$ and changes in the level of the oil free surface $(z)$ along the radius $(r)$ of compartment A of the oil bath with its shown above parameters and hydraulic unit operation mode. The calculations show that with this design of the oil bath, the oil fill level of $915 \mathrm{~mm}$ and hydraulic unit rotation speed of $200 \mathrm{rpm}$, the free surface curve in compartment A wedges out onto its ceiling, thereby creating a potential corresponding to $740 \mathrm{~mm}$ liquid (oil) column. This potential is realized in the oil overflow through the 12 upper holes in the rotor hub from compartment A to compartment $\mathrm{B}$, as well as into the oil discharge towards the side of the hub when the unit is gaining speed. It can be seen that the shell flange at the level $z=1063 \mathrm{~mm}$ is touched by the free surface of the oil. The latter creates the conditions for entering the oil on the stationary shell flange with further release of oil through the shell on the shaft of the hydraulic unit. 


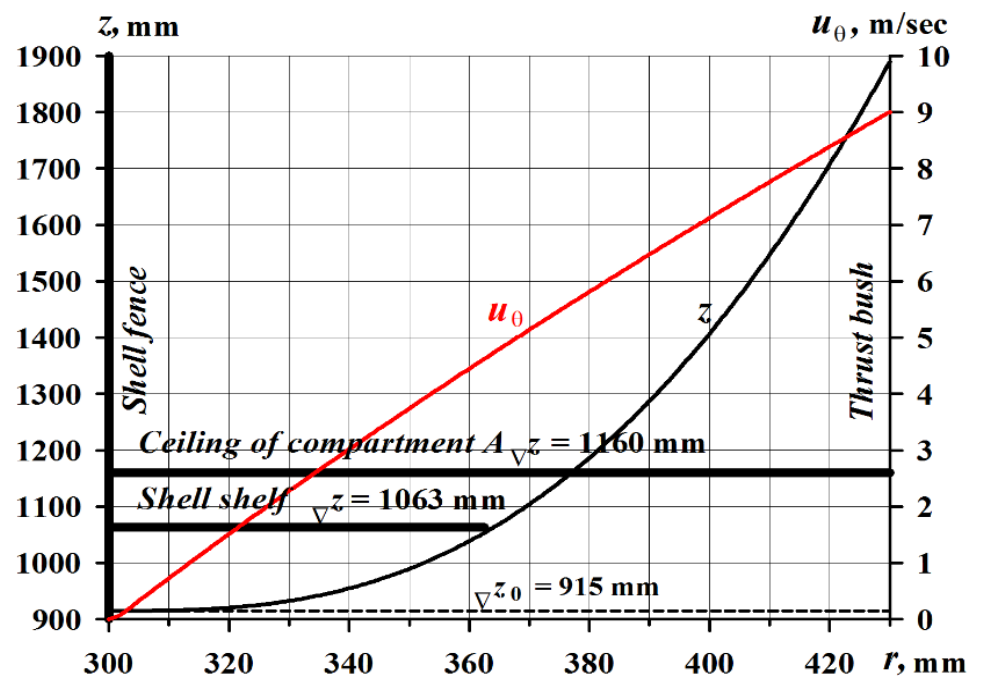

Fig. 3. Azimuthal velocity distribution and piezometric line, corresponding to the oil free surface in the area up to the ceiling of compartment $\mathrm{A}$ of the standard oil bath of the thrust bearing of the Bajo de Mina HPP generator.

Meanwhile, it must be emphasized that the exit of the oil free surface to the ceiling of compartment $\mathrm{A}$ in the area adjacent to the rotating bush does not pose a danger in general with the steady-state mode of movement associated with the release of oil through the shell onto the hydraulic unit generator shaft. For in this case, the oil rotates together with the bush and is pressed against its surface by centrifugal forces. Wedging out of the oil free surface on the ceiling of compartment A is dangerous only during the start-up of the hydraulic unit, when the oil starts to spin up from rest position with the rotating bush. During this period, the free surface transforms from horizontal to curvilinear in the conditions of a rapid increment in the oil level in the area adjacent to the bush. Therefore, during this period, the oil has a significant vertical speed along the surface of the bush. It is this vertical speed, taking into account the configuration of the smoothly connecting radius of the bush shaft with the ceiling of compartment A (cf. Fig. 1,a), as a result, it can be realized in the ejection of oil towards the shell.

This can be avoided by reducing the thickness of the rotating layer in compartment A by installing an additional shell (item 8 in Fig. 1,b) between the main shell (item 2 in Fig. 1,a) and the bush (item 4 in Fig. 1,a ), as well as by implementing the measures described in subsection 3.1 above. As a result of the changes, the modernized oil bath became in line with that accepted for execution at Bajo de Mina HPP (cf. Fig. 1,b). The parameters of the upgraded oil bath, taken into account in further calculations: the radius of the additional shell $R_{1}=362.5 \mathrm{~mm}$, installation height above the bottom of the oil bath of the perforated ring (item 9 in Fig. 1,b) at $z=625 \mathrm{~mm}$, oil fill level $895 \mathrm{~mm}$. These parameters correspond to the condition $z=0.3 R_{2}=0.3 \cdot 430=129 \mathrm{~mm}$, which is significantly less than the depth of the oil mass in the modernized compartment $\mathrm{A}$, equal to $895-625=270 \mathrm{~mm}$. This allows you to use equations (23) and (24) written in the absolute values of the variables when calculating the parameters of oil movement in compartment A of the modernized oil bath.

Fig. 4 shows the calculated relation curves of distribution of azimuthal velocities $\left(u_{\theta}\right)$ and changes in the level of the oil free surface $(z)$ along radius $(r)$ of the modernized compartment A of the oil bath with the indicated parameters and the rated speed of $t$ rotation of the hydraulic unit shaft $-450 \mathrm{rpm}$. 


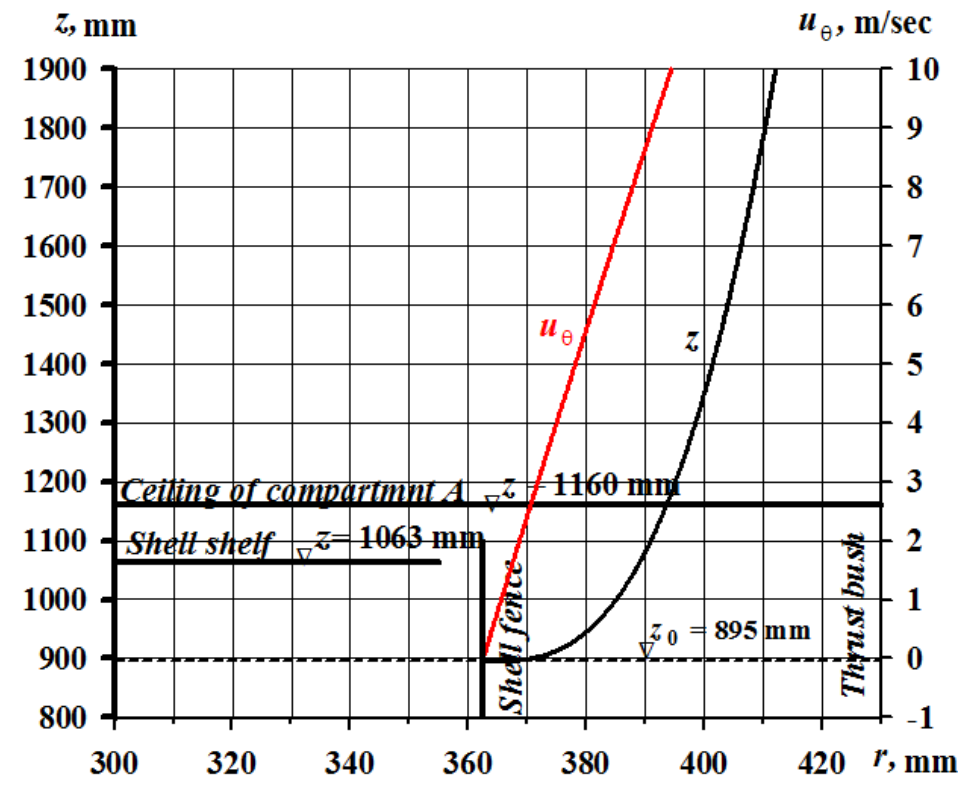

Fig. 4. Azimuthal velocity distribution and piezometric line corresponding to the oil free surface in the area to the ceiling of compartment A of modified oil bath of the Bajo de Mina HPP generator thrust bearing.

You can see that the situation in compartment A of the modernized oil bath (cf. Fig. 4) has fundamentally changed for the better compared to the situation in the standard oil bath (cf. Fig. 3). The oil free surface is removed for a distance of $30 \mathrm{~mm}$ from the window in the additional shell located under the ceiling of the modernized compartment A. This is guaranteed to prevent oil from entering the shell flange and, especially, the generator shaft. Thus, the set task - to eliminate the release of oil through the shell of the oil bath of the thrust bearing on the shaft of the Bajo de Mina HPP generator is solved.

As a side measure, it was implemented: by permanent additionally blowing the air through the gap (item 3 in Fig. 1,a) between the standard shell (item 2 in Fig. 1,a) and the generator shaft (item 1 in Fig. 1,a) increase in the modernized compartment A of the gauge pressure by $186 \mathrm{~Pa}$. This prevents oil mist (oil vapor) from entering the gap and onto the generator shaft.

After the modernization on October 4, 2011, unit No.2 was commenced. At start-up the rated speed of $450 \mathrm{rpm}$ has been attained. The generator worked at the rated speed for 3 hours 18 minutes until steady-state temperatures were obtained. No oil emissions have been observed.

\section{Conclusions}

1. The purpose of the work is to determine the causes of oil overflow through the thrust bearing oil bath shell of the Bajo de Mina HPP (Panama) hydro generator and to analyze possible ways to remedy the occurring emergency situation.

2. As a result of the work, an analytical model was obtained and verified for calculating the distribution of azimuthal velocities and the shape of the oil free surface in the compartments of the oil bath of the vertical-shaft unit thrust bearing.

3. It has been established that the oil flow in compartment A of the oil bath is independent of viscosity and Reynolds number. Physical modeling of such flows should be performed according to the Froude criterion. 
4. In compartment $\mathrm{A}$, the inhibitory effect of the bottom ceases to affect the azimuthal fluid velocities diagram when moving away from the bottom for a distance exceeding 0.3 of the inner radius of the bush. The radial distribution of azimuthal velocities in compartment $\mathrm{A}$ far from the bottom is close to a linear law.

5. The radially increasing oil free surface in compartment $\mathrm{A}$ has a profile close to parabolic configuration.

6. The calculations showed that with the design of the oil tank as of 08.08.2011 (before modernization), oil fill level of $915 \mathrm{~mm}$ and generator rotation speed of $200 \mathrm{rpm}$, the free surface curve in compartment A wedges out onto its ceiling, thereby creating a potential corresponding to $740 \mathrm{~mm}$ column of fluid (oil). This potential is realized in the oil flow through 12 upper holes in the thrust bush, $25 \mathrm{~mm}$ in diameter, from compartment A to compartment $\mathrm{B}$, as well as into the oil discharge towards the shell. The latter creates the conditions for oil entering the shell flange with the further oil release to the shaft of the unit

7. The significant potential for oil release to the ceiling of compartment A shows that the measures restricted only by decrease in the "rest level" are insufficient to obtain the positive effect associated with suppressing the release of oil onto the generator shaft.

8. The means to prevent the release of oil through the shell on the generator shaft consist in reducing the thickness of the rotating layer. This is achieved by installing an additional shell located between the standard shell and the hub. The proposal to install such an additional shell made by the Electrotyazhmash plant is one of the main ones in solving the problem under consideration and remedying the emergency situation.

9. The essential solutions consist in: installing a vertical annular perforated shield on the bearing housing (item 10 in Fig. 1,b) that covers compartment $\mathrm{C}$ from the rotating hub and installing a horizontal perforated locking ring in compartment A (item 9 in Fig. 1,b ) In this case, there will be no circulating movement of oil in compartment $\mathrm{C}$ and, therefore, there will be no possibility of dangerous dynamics when twisting the overwhelming mass of oil located in compartment $\mathrm{C}$ of the oil bath of the thrust bearing and guide bearing of the Bajo de Mina HPP generator.

10. The system of air blowing into the casing under the thrust bearing oil bath was modernized with an additional nine pipes of $100 \mathrm{~mm}$ in diameter that allowed satisfactory ventilation of the oil bath compartments.

11. The calculations confirm and justify the need, feasibility and sufficiency of all measures to upgrade the primary version of the oil bath of the Bajo de Mina HPP generator to eliminate the emergency situation associated with the release of oil onto the unit shaft. This was confirmed by the commissioning tests on October 4, 2011 and the subsequent 9 year period of operation of the power plant.

\section{Acknowledgements}

The author are grateful to Ph.D, Advisor to the General Director of "Energomashexport Corporation" LLC Shamil Ignatievich Abubakirov for his suggestion to conduct the research reported here.

\section{References}

1. E. Aleksandrov, Thrust bearings (EDP Energy, Moscow, 1975)

2. O.V. Antonova, Yu.Ya. Boldyrev, A.I. Borovkov, I.B. Voinov, J. Mach. Manufacture and Reliability 46, 6 (2017)

3. M. Wasilczuk, Lubr. Sci. 3, 2 (2015)

4. M. Wasilczuk, M. Wodtke, L. Dąbrowski, Encyclopedia of Tribology (EDP Springer 
Science+Business Media, New York, 2013)

5. D.M.C. McCarthy, S.B. Glavatskih, A. Byheden, Lubr. Sci. 21 (2009)

6. A.L. Zuikov, Hydrodynamics of circulating flows (EDP ACB, Moscow, 2010)

7. G. Rotta, M. Wasilczuk, Tribol. Int. 41 (2008)

8. M. Wodtke, A. Schubert, M. Fillon, M. Wasilczuk, P. Pajączkowski, J. Eng, Tribol. 228 (2014)

9. M. Wasilczuk, G. Rotta, Tribol (2013)

10. O.V. Antonova, Yu.Ya. Boldyrev, A.I. Borovkov, I.B. Voinov, Mater. Phys. and Mech. 34, 1 (2017)

11. A. Ettles, J. Seyler, M. Bottenschein, Tribol. Transactions 48, 4 (2005)

12. Y. Peng, X.Y. Chen, K.W. Zhang, G.X. Hou, J. Hydrod. 19, 5 (2007)

13. Y. Xu, Z.H. Li, X.D. Lai, 2011 IEEE Conf. (2011)

14. X. D. Lai, G. L. Liao, Y. Zhu, X. Zhang, Q.Q. Gou, W.B. Zhang, 26 IAHR Symp. 15 (2012)

15. G.C. Brito Junior, R.D. Machado, A.C. Neto, M.F. Martini, Int. J. Rot. Mach. (2017)

16. M. Wasilczuk, M. Wodtke, L. Dąbrowski, Lubr. Sci. 5, 4 (2017)

17. T.H. McCloskey, Proc. 24 Turbomach. Symp. (2005)

18. M.G. Mikhailov, Unforeseen situations in the construction of hydropower plants (EDP NIPKTS Voskhod-A, Moscow, 2016)

19. M.G. Mikhailov, Hydrotechnical Construction 6, 33-38 (2015)

20. M.G. Mikhailov, Hydrotechnical Construction 1 (2016)

21. M.G. Mikhailov, Hydrotechnical Construction 5 (2017)

22. M.G. Mikhailov, Hydrotechnical Construction 12 (2017)

23. G.A. Korn, T.M. Korn, Mathematical Handbook for Scientists and Engineers: Definitions, Theorems and Formulas for Reference and Review (EDP Dover Publications, New York, 2000)

24. G. K. Batchelor, An Introduction to Fluid Dynamics (EDP University Press, Cambridge, 2005)

25. N.I. Zubarev, V.A. Spektor, V.A. Belyh, N.A. Korneev, E.V. Vangalov, R.B. Petuhov, E.C. Daribazaron, The study of the movement of oil in the bath of the thrust bearing of the hydrogenerator in order to clarify the influence of individual factors on the shape of the free surface of the oil (EDP VNIIG B.E. Vedeneev, Leningrad, 1987) 